\title{
Robust and high-speed rotation control in optical tweezers by using polarization synthesis based on heterodyne interference
}

\author{
Wei Liu ${ }^{1,2}$, Dashan Dong1,2, Hong Yang1,2,3, Qihuang Gong1,2,3 and \\ Kebin Shi1,2,3*
}

\begin{abstract}
The rotation control of particles in optical tweezers is often subject to the spin or orbit angular momentum induced optical torque, which is susceptible to the mechanical and morphological properties of individual particle. Here we report on a robust and high-speed rotation control in optical tweezers by using a novel linear polarization synthesis based on optical heterodyne interference between two circularly polarized lights with opposite handedness. The synthesized linear polarization can be rotated in a hopping-free scheme at arbitrary speed determined electronically by the heterodyne frequency between two laser fields. The experimental demonstration of a trapped vaterite particle in water shows that the precisely controlled rotation frequency of $300 \mathrm{~Hz}$ can be achieved. The proposed method will find promising applications in optically driven micro-gears, fluidic pumps and rotational micro-rheology.
\end{abstract}

Keywords: polarization modulation; optical heterodyne; optical tweezers; optical rotation

Liu W, Dong D S, Yang H, Gong Q H, Shi K B. Robust and high-speed rotation control in optical tweezers by using polarization synthesis based on heterodyne interference. Opto-Electron Adv 3, 200022 (2020).

\section{Introduction}

Manipulation of particle rotation in optical tweezers has found many applications such as viscosity sensing, fluidic pumping $^{1-4}$ and photo-mechanics ${ }^{5-7}$. The angular momentum of circularly polarized or vortex laser field can be transferred to trapped object and result in an optical torque for rotating object by scattering or absorbance ${ }^{5,8-11}$. For a birefringent or deformed particle trapped in viscous fluid, rotation with certain frequency can be initiated by the counteracting between optical angular momentum induced torque and the resistive torques induced by the ambient ${ }^{12}$. As a result, the particle rotation control driven by optical angular momentum is often susceptible to various factors including the intensity of light ${ }^{13}$, the morphology of particle, and the viscosity coefficient of the ambient. Rotation speed calibration in angular momentum based optical tweezers therefore is usually required for precisely controlling the rotation speed ${ }^{3,14-16}$.

In contrast to the use of circularly polarized or vortex beam, the torque induced by the misalignment between the linearly polarized laser field and optical axis of the trapped particle is insusceptible to ambient viscosity and therefore can generate synchronized particle rotation with the rotating linear polarization ${ }^{17-19}$. However, it is still limited in achieving particle rotation based on linear polarization modulation in optical tweezers that are continuous, hopping-free, and high-speed simultaneously. For examples, mechanically rotating half-wave plate is the most straightforward method which can continuously rotate the polarization plane of the beam while the rotational rate of this method is slow due to the mechanical

\footnotetext{
${ }^{1}$ State Key Laboratory for Mesoscopic Physics and Frontiers Science Center for Nano-optoelectronics, School of Physics, Peking University, Beijing 100871, China. ${ }^{2}$ Collaborative Innovation Center of Extreme Optics, Shanxi University, Taiyuan 030006, China. ${ }^{3}$ Peking University Yangtze Delta Institute of Optoelectronics, Nantong 226010, China.

${ }^{*}$ Correspondence: K B Shi, E-mail: kebinshi@pku.edu.cn

Received: 11 July 2020; Accepted: 15 July 2020; Published: 21 August 2020
} 
movement $^{17,20}$. The polarization direction can also be rotated electrically by changing the phase difference of the vertical and the horizontal components of the input polarization using a pair of acoustic-optical modulators $(\mathrm{AOMs})^{21}$. Yet this method can only generate rotational polarization positioning in discrete manner and the discontinuity of the polarization rotation hinders its application in high-speed rotation manipulation. Quasi-continuous rotation of polarization plane can be achieved by using electro-optic modulators (EOMs) or photo-elastic modulators (PEMs) in which the polarization angle of the output beam is proportional to the phase modulation driven by the control voltage of the modulators $^{22,23}$. The dynamic range of linear phase modulation of the EOMs or PEMs is usually limited. Therefore, EOMs or PEMs based linear polarization control is limited by a wrapped phase modulation, making it difficult to generate truly hopping-free rotation of linear polarization plane.

By using a novel linear polarization synthesis based on optical heterodyne interference between two circularly polarized lights with opposite handedness, we report on a robust and high-speed linear polarization rotation control method. The synthesized linear polarization can be rotated unidirectionally at arbitrary speed determined electronically by the heterodyne frequency between two laser fields. High-speed rotation manipulation of single birefringent particle in optical trapping systems using the synthesized linearly polarized light was also experimentally demonstrated. The particle rotation speed was demonstrated to be synchronized to the laser heterodyne frequency and only limited by the optical torque induced by the maximum laser power.

\section{Theory and experiments}

A linearly polarized light can be expressed as the superposition of a right-handed circularly polarized light and a left-handed circularly polarized light as shown in below :

$$
E_{0}=\cos \theta \hat{\boldsymbol{x}}+\sin \theta \hat{\boldsymbol{y}}=\frac{1}{\sqrt{2}} \mathrm{e}^{\mathrm{i} \theta}\left(\hat{L}+\mathrm{e}^{-\mathrm{i} 2 \theta} \hat{R}\right) \quad,
$$

where the basis vector of left-handed circularly polarized light and right-handed circularly polarized light can be expressed by $\hat{L}=1 / \sqrt{2}(\hat{\boldsymbol{x}}+\mathrm{i} \hat{\boldsymbol{y}})$ and $\hat{R}=1 / \sqrt{2}(\hat{\boldsymbol{x}}-\mathrm{i} \hat{\boldsymbol{y}})$ respectively, the $\hat{x}, \hat{y}$ are the basis vectors in Cartesian coordinate. $\theta$ is the polarization angle with respect to the $x$ axis given that the phase difference between the two orthogonal circularly polarized beams is $2 \theta$ as shown in Fig.1(a). In a heterodyne interference arrangement where the laser frequencies of the left-handed circularly polarized light and the right-handed circularly polarized light are different, the synthesized polarization can be expressed as:

$$
\begin{aligned}
E_{0}(t) & =\mathrm{e}^{-\mathrm{i}\left(2 \pi f_{1} t+\varphi_{1}\right)} \hat{R}+\mathrm{e}^{-\mathrm{i}\left(2 \pi f_{2} t+\varphi_{2}\right)} \hat{L} \\
& =\mathrm{e}^{-i 2 \pi f_{1} t+\varphi_{1}}\left(\hat{R}+\mathrm{e}^{-i\left[2 \pi\left(f_{2}-f_{1}\right) t+\varphi_{2}-\varphi_{1}\right]} \hat{L}\right),
\end{aligned}
$$

where $f_{1}$ and $f_{2}$ are the frequencies of two beams respectively; $\varphi_{1}$ and $\varphi_{2}$ are the initial phase of two beams. As shown in equation (2), the phase difference between the two beams at time $t$ can be expressed as $2 \pi\left(f_{2}-f_{1}\right) t+\varphi_{2}-\varphi_{1}$, where $\varphi_{2}-\varphi_{1}$ is constant and determined by the initial phase of the two beams. The item $e^{-\mathrm{i} 2 \pi f_{1} t+\varphi_{1}}$ represents the carrier phase and has no effect on the modulation of the polarization plane. Comparing equation (2) to equation (1), the time dependent linear polarization angle can be expressed as :

$$
\theta(t)=\frac{2 \pi\left(f_{2}-f_{1}\right) t+\varphi_{2}-\varphi_{1}}{2}
$$

If $\left|f_{1}-f_{2}\right|<<f_{1}$, noting that the modulated linear polarization rotation is periodical at the angle of $\pi$, the continuous rotation of the polarization over time then is at the frequency of $f_{2}-f_{1}$. Furthermore, the accumulated phase is monotonously increasing and therefore leads to a hopping-free polarization modulation. Fig. 1(b) shows

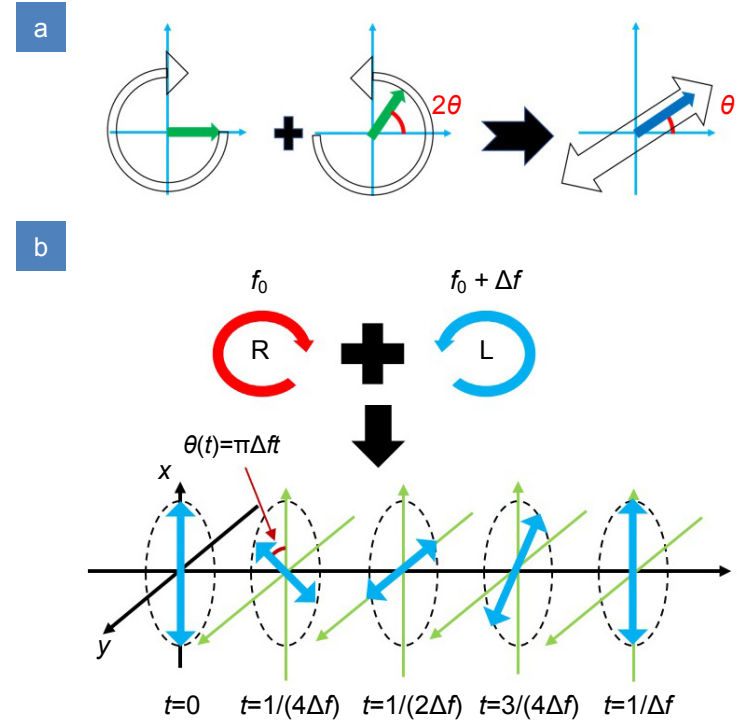

Fig. 1 | Design principle of generating a linearly polarized beams with a rotating polarization angle based on optical heterodyne interference. (a) Linearly polarized beam with angle $\theta$ to the $x$-axis can be generated by superposition of two circularly polarized beams with opposite handedness and the phase difference of $2 \theta$. (b) Linearly polarized beam with a rotating polarization angle by superposition of two circularly polarized beams with opposite handedness. The polarization angle rotated at frequency of $\Delta f / 2$, where $\Delta t$ is the heterodyne frequency of two laser fields. 
the schematic spinning dynamics of the polarization plane with laser heterodyne frequency of $\Delta f=f_{2}-f_{1}$ in one heterodyne period.

The schematic diagram of the experiment is shown in Fig. 2. A pair of acoustic-optical modulator was controlled by a dual-channel RF (radio frequency) drive circuit and a pair of acousto-optic crystals (Chongqing Shangmao Technology Development Co. Ltd). The central working frequency of the two acousto-optic crystal is $99 \mathrm{MHz}$ with frequency stability of $\pm 0.75 \mathrm{~Hz}$ while the RF of one channel is adjustable within range from $98 \mathrm{MHz}$ to 99 $\mathrm{MHz}$ with resolution of $10 \mathrm{~Hz}$. Because the phase of the beams diffracted by AOMs is affected by the phase of input RF signals, it is necessary to drive two AOMs with RF circuits referenced to the same clock oscillator, which can ensure the coherence and phase stability of two beams. A $532 \mathrm{~nm}$ single longitudinal mode laser with a coherence length of $50 \mathrm{~m}$ was chosen as the light source for obtaining stable optical heterodyne interference. The laser first passed a collimating system composed of two confocal lenses to make the beam waist of the beam matching with the aperture diameter of the AOMs. A half-wave plate and a polarized beam splitter were used to split the beam with adjustable power distributions. The two orthogonally polarized beams passed through AOMs in each arm and then were combined by another polarized beam splitter. The two beams were frequency shifted to $f_{0}+\Delta f_{1}$ and $f_{0}+\Delta f_{2}$ respectively, where $f_{0}$ is the frequency of incoming beam; $\Delta f_{1}$ and $\Delta f_{2}$ is the frequency shift induced by the two AOMs. A quarter wave plate was used to transfer two beams into oppositely handed circularly polarized light after beam recombination with the $45^{\circ}$ angle relative to the horizontal polarization direction. The rotation frequency of the linear polarization direction is then given as:

$$
\Delta f=\Delta f_{1}-\Delta f_{2} .
$$

The beam waist of the output linearly polarized beam was expanded to $6 \mathrm{~mm}$ by a beam expander made of an objective and a plano-convex lens. The beam was then reflected by a $561 \mathrm{~nm}$ long-pass dichroic mirror (DM) to the back focal plane of the objective lens and formed optical trapping after being focused by the objective with numerical aperture (NA) of 1.4. The DM was customized in its coating for minimizing the depolarization effect in reflection. The trapped particles were illuminated with Kohler illumination and imaged with an infinity calibrated imaging system. The rotational dynamics of the particles can be directly characterized by the camera in the case of slow rotation. A horizontally polarized probe laser of $671 \mathrm{~nm}$ wavelength was used to detect the rotation frequency by logging the scattering fluctuation of rotating particle with a photodetector $(\mathrm{PD})$ at the vertical polarization direction. The time-lapsed scattering signal can be used to determine the particle rotation speed.

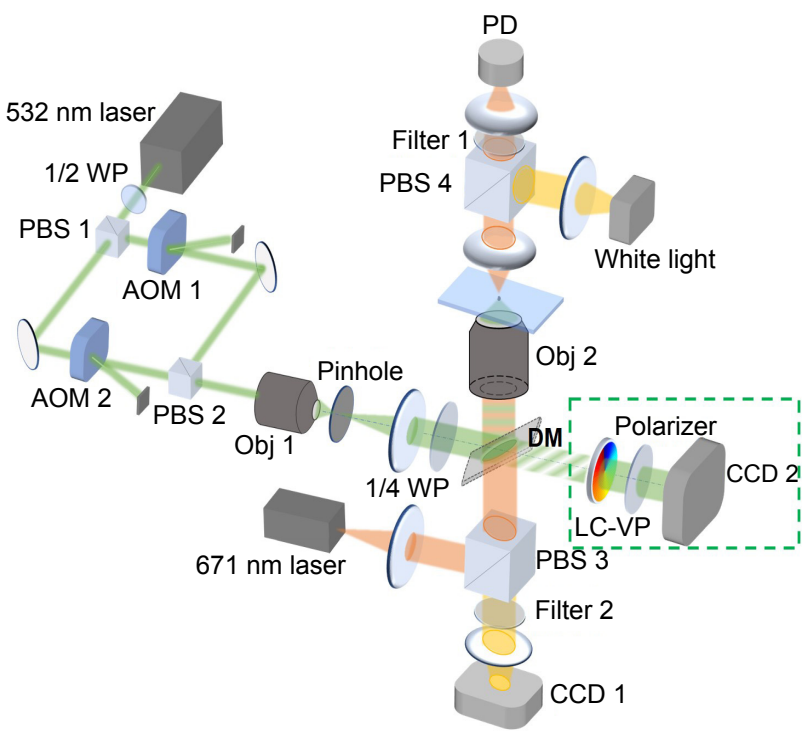

Fig. 2 | Schematic diagram of the experiment. The trapping beam is produced by a $532 \mathrm{~nm}$ laser and a Mach-Zehnder interferometer based on heterodyne interference. Devices in the green dashed box: a polarization detector made of a liquid crystal vortex half wave-plate and a polarizer which determines the polarization angle of the output trapping field. WP: wave plate; DM: $561 \mathrm{~nm}$ long pass dichroic mirror mounted on a flippable frame; PD: photodiode; PBS 1-4: polarized beam splitter; LC-VP: Liquid crystal based vortex half-wave plate; Obj 1: 10x objective; Obj 2: 100x objective with NA of 1.4; Filter 1-2: $635 \mathrm{~nm}$ long-pass filter.

\section{Results and discussion}

To visualize the rotation of the linear polarization synthesized, a passive liquid crystal vortex half-wave plate (LC-VP) combined with a polarizer was used to detect the polarization direction of linearly polarized beam ${ }^{24,25}$ as shown in the green dashed box in Fig. 2. The vortex half-wave plate can convert linearly polarized light into a cylindrical vector beam ${ }^{26}$. As a result, a rotating linearly polarized light becomes a dynamic cylindrical vector beam switching between radially and azimuthally polarized beams continuously. Assuming heterodyne frequency set to $\Delta f$, the phase difference of the two beams will be a function of time written as $2 \pi \Delta f t$. The light field passing through the vortex half-wave plate at time $t$ can be described as a Jones vector in Cartesian coordinates: 


$$
\begin{aligned}
\boldsymbol{E}_{\mathrm{Cylin}} & =\left(\begin{array}{c}
\cos (\pi \Delta f t+\varphi) \\
\sin (\pi \Delta f t+\varphi)
\end{array}\right) \\
& =\cos (\pi \Delta f t) E_{\mathrm{RP}}+\sin (\pi \Delta f t) E_{\mathrm{AP}},
\end{aligned}
$$

where $\varphi$ is the azimuth angle around the axis of the beam; $\boldsymbol{E}_{\mathrm{RP}}$ and $\boldsymbol{E}_{\mathrm{AP}}$ are Jones vectors for the radically or azimuthal polarized light field respectively which are written $\operatorname{as}^{27-30}$ :

$$
\begin{aligned}
& \boldsymbol{E}_{\mathrm{RP}}=\left(\begin{array}{l}
\cos (\varphi) \\
\sin (\varphi)
\end{array}\right) \\
& \boldsymbol{E}_{\mathrm{AP}}=\left(\begin{array}{l}
\cos (\varphi+\pi / 2) \\
\sin (\varphi+\pi / 2)
\end{array}\right)
\end{aligned}
$$

Figure 3(a) shows simulated field distributions of cylindrical vector light at time interval of $T / 4$, where $T$ is beat period that equals to $1 / \Delta f$. When the cylindrical vector light passes through a polarizer, a two-lobed intensity distribution is formed as the simulated result shown in Fig. 3(b), where the heterodyne frequency was set to $125 \mathrm{~Hz}$ in simulation. In the experiment, the heterodyne frequency was also set at $125 \mathrm{~Hz}$. Since the cylindrical vector beam switches between radial and azimuthal polarizations every 180 degrees of rotation of input linear polarization direction, it can be seen that the rotational frequency of the two-lobed structure is equal to the heterodyne frequency. The experimental observation shown in Fig. 3(c) indicates that a dynamic light field with continuously rotating linear polarization plane is generated at heterodyne frequency of $125 \mathrm{~Hz}$, agreeing well with the theoretical design.

The modulated linear polarization then was used to achieve particle rotation in optical tweezers. Vaterite particles with $1 \mu \mathrm{m}$ diameter suspending in distilled water were used in the optical trapping experiment. Vaterite is a poly-crystalline structure of calcium carbonate consisting of 20-30 nm nanocrystals ${ }^{31-33}$ with positive uniaxial birefringence. The alignment of the optical axis of the nanocrystals has a hyperbolic structure which makes vaterite highly anisotropic. The birefringence coefficient of vaterite is up to $0.1^{12,34,35}$. Linearly polarized light can be used to control the optical axis orientation of vaterite particles effectively, making it an excellent candidate for optical rotation manipulation and angular momentum transport. When a vaterite particle is trapped by a linearly polarized beam, the effective torque can be expressed by $^{33}$ :

$$
\tau=-\frac{\varepsilon}{4 \pi f_{0}} S E_{0}^{2} \sin \left[k_{0} d\left(n_{\mathrm{o}}-n_{\mathrm{e}}\right)\right] \sin 2 \theta \quad,
$$

where $S$ is the particle cross-section area, $\varepsilon$ is the permit-
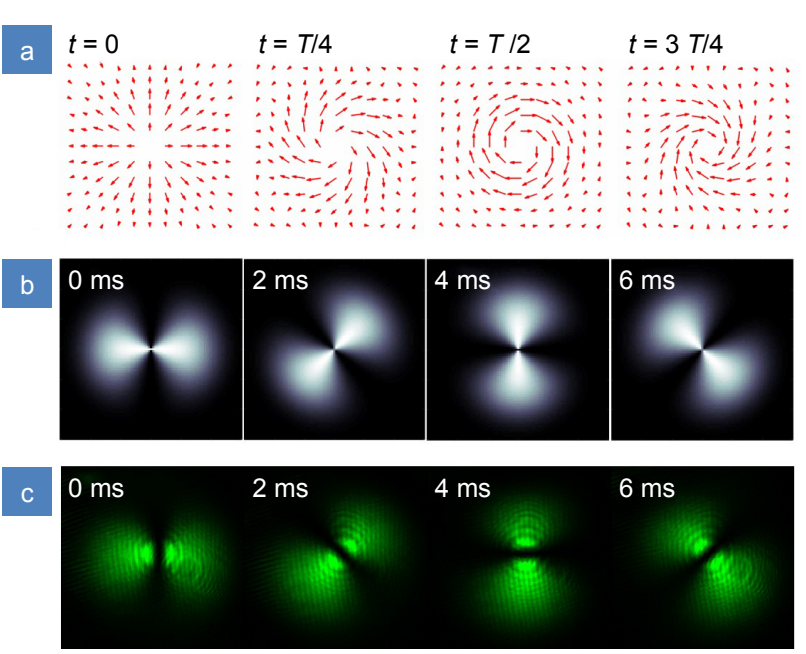

Fig. 3 | Verifying the polarization orientation distribution after passing through a LC-VP device. (a) Polarization angle distributions every $T / 4$, where $T$ is beat period that equals to $1 / \Delta f$. (b) The simulated light-field intensity distributions of the output trapping field recorded after passing the polarization detector consisting of a liquid crystal vortex half wave-plate and a polarizer when heterodyne frequency was set to $125 \mathrm{~Hz}$. (c) The experimental light-field intensity distributions recorded by a camera confirming that the polarization angle of the output trapping light rotated counterclockwise corresponding to (b) (Supplementary Video 1).

tivity, and $f_{0}$ is the frequency of input light field. In the first sinusoidal term in equation (7), $n_{\mathrm{o}}$ and $n_{\mathrm{e}}$ are optical indices along the ordinary axis and extraordinary axis, respectively, $d$ is the thickness of the particle, and $k_{0}$ is the wave number of the laser beam in vacuum. $\theta$ is the offset angle between the linear polarization direction of the beam and the optic axis of particle in the second sine term.

The synthesis of vaterite particles was based on the modification of a previously published protocol ${ }^{33,34}$. Aqueous solutions of $\mathrm{CaCl}_{2}, \mathrm{~K}_{2} \mathrm{CO}_{3}$, and $\mathrm{MgSO}_{4}$ were prepared which all have a molarity of $0.1 \mathrm{M}$. And a $5 \mathrm{~mL}$ plastic vial was used as reaction vessel. First, $1.5 \mathrm{~mL}$ of $\mathrm{CaCl}_{2}$ solution and $60 \mu \mathrm{L}$ of $\mathrm{MgSO}_{4}$ solution were pipetted into the vial, followed by surfactant solution (XYS-3500, Yancheng Yunfeng Chemical Co., Ltd) with dilution ratio of 1:1000 and then $90 \mu \mathrm{L}$ of $\mathrm{K}_{2} \mathrm{CO}_{3}$ solution. The solution was agitated by pipetting of the solution with a plastic dropper. Disc-shaped vaterite particles solution was obtained after 5 minutes of pipetting.

Figure 4(a) shows the top view of a disc-shaped vaterite particle lying on the substrate, where its optical axis is perpendicular to the disc surface. After being optically trapped, the disc-shaped vaterite particle tends to align its optical axis with the orientation of the incoming linear polarization. As a result, the trapped particle flips $90^{\circ}$ 


\section{Opto-Electronic Advances https://doi.org/10.29026/oea.2020.200022}

under laser trapping and its side facet can be imaged as shown in Fig. 4(b). The trapped particle exhibits two-fold rotational symmetry in the camera image. When the particle rotates at a relative low speed, a high-speed camera can be used to directly visualize the rotation dynamics. Figure 4(c) shows the rotation process of a vaterite particle with diameter of $1.5 \mu \mathrm{m}$ and thickness of $1 \mu \mathrm{m}$ captured by rotating linearly polarized light with power of 50 $\mathrm{mW}$. The heterodyne frequency was tuned to $95.4 \mathrm{~Hz}$ with nominal frequency uncertainty of $\pm 0.75 \mathrm{~Hz}$. The actual rotation frequency of the particles measured by the camera is $47.6 \mathrm{~Hz}$ which agrees well with the theoretical rotation frequency of $47.7 \mathrm{~Hz}$.

In order to systematically study the applicability and control accuracy of the proposed method for high-speed rotation operation, the heterodyne frequency was adjusted from $100 \mathrm{~Hz}$ to $600 \mathrm{~Hz}$ at intervals of $100 \mathrm{~Hz}$ for a smaller vaterite particle with a diameter of $1 \mu \mathrm{m}$ and a thickness of $0.7 \mu \mathrm{m}$. Fig. 5(a) shows the temporal scattering signal of the particles recorded by the photodiode on
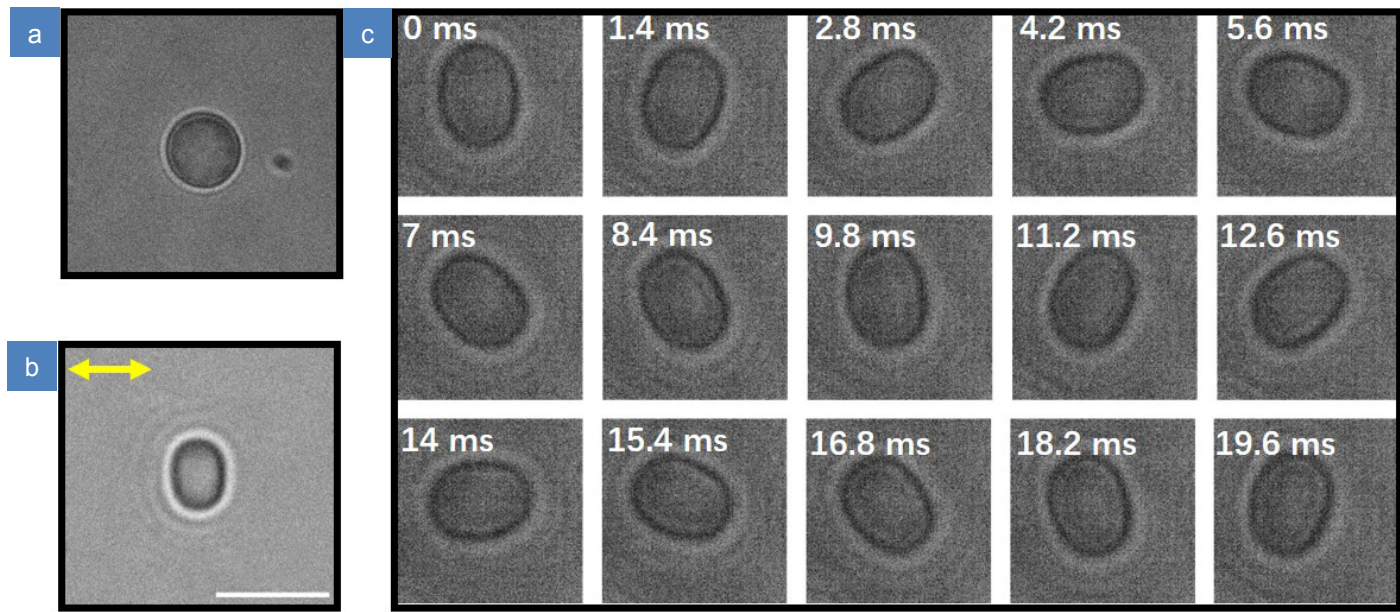

Fig. 4 | Video snapshots of disc-shaped vaterite particles recorded by optical tweezer apparatus. (a) Top view of a vaterite particles before being trapped. Note that the optical axis is perpendicular to the top facet. (b) Top view of the vaterite particles after being trapped by the linear polarized beam with the polarization angle indicated by the yellow arrow. (c) Video images recorded every $1.4 \mathrm{~ms}$ for a $1.5 \mu \mathrm{m}$ diameter and 1 $\mu \mathrm{m}$ thickness vaterite micro-disc being rotated clockwise in the optical trap (Supplementary Video 2).
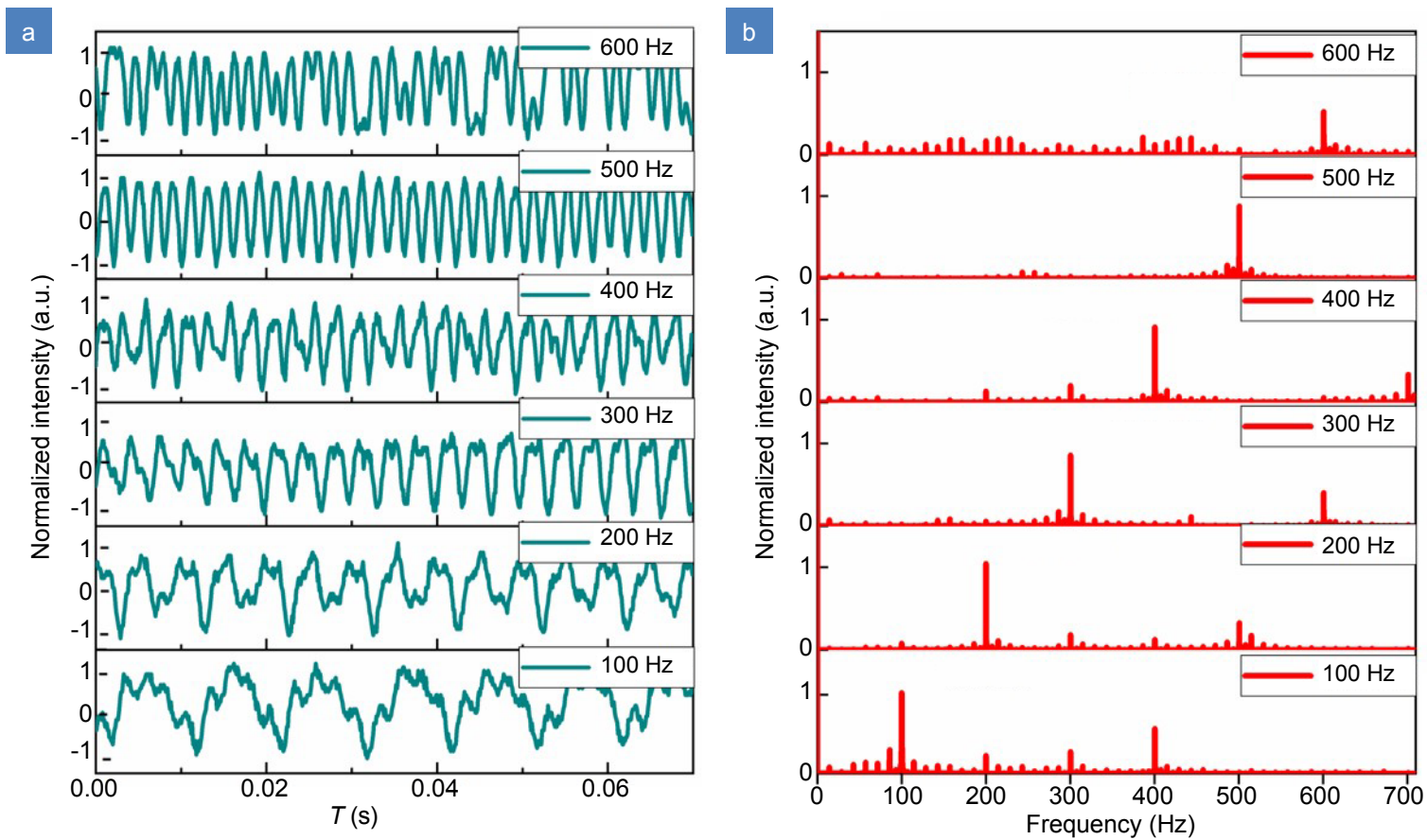

Fig. 5 | The scattered light signals of one trapped vaterite particle. (a) The signals of the probe laser scattered by rotating vaterite particle at heterodyne frequency tuned with the step of $100 \mathrm{~Hz}$. (b) The frequency spectra obtained by Fourier transform to the signals in (a). 
the probe light. The scattering signal of vaterite was recorded for 4.47 seconds at the sampling rate of 10000 points/s. Fourier transform was performed on the data sets to obtain the frequency spectrum as shown in Fig. 5(b). As the temporal waveform shown in Fig. 5(a) is not ideal sinusoidal distributions, minor harmonic frequencies can be observed in Fig. 5(b). Since the particles have a two-fold rotational symmetry, it can be observed in Fig. 5 that the frequency of the scattered signal agrees well with the optical heterodyne frequency. Yet the actual 360-degree-rotation frequency of the particles should be the half of the measured frequency. Fig. 5(b) indicates that the particles rotational rate still maintains good speed accuracy even at a high rate of $600 \mathrm{~Hz}$ in aqueous solution. It should be noted that there is still a threshold for maximum rotation speed of the trapped particle determined by laser power and viscosity of the ambient medium.

\section{Conclusions}

In summary, we propose a novel method for hopping-free rotation of linear polarization by electronically tuning the laser heterodyne interference, showing its promising applications in robust and high-speed particle rotation manipulation in optical tweezers. The modulation speed and stability of generated rotating linear polarization is only limited by the AOM detuning frequency range and the resolution of the driving RF source. Therefore, it can potentially reach $\mathrm{MHz}$ scale with sub- $\mathrm{Hz}$ accuracy. High speed rotation of vaterite particles synchronized to polarization modulation is demonstrated in optical tweezers. The reported rotation control in optical trapping will find important applications in on chip micro-pumping, viscosity sensing, rotational manipulation in biophysical studies, and measurements of torque ${ }^{1,36-38}$. It is also anticipated that the proposed polarization modulation method will benefit general research fields such as polarization sensitive imaging and spectroscopy ${ }^{39-46}$.

\section{References}

1. Leach J, Mushfique $H$, di Leonardo R, Padgett M, Cooper J. An optically driven pump for microfluidics. Lab Chip 6, 735-739 (2006).

2. Ladavac K, Grier D G. Microoptomechanical pumps assembled and driven by holographic optical vortex arrays. Opt Express 12, 1144-1149 (2004).

3. Ahn J, Xu Z J, Bang J, Ju P, Gao X Y et al. Ultrasensitive torque detection with an optically levitated nanorotor. Nat Nanotechnol 15, 89-93 (2020).

4. Zhu J M, Zhu X Q, Zuo Y F, Hu X J, Shi Y et al. Optofluidics: the interaction between light and flowing liquids in integrated devices. Opto-Electron Adv 2, 190007 (2019).

5. Beth R A. Mechanical detection and measurement of the angular momentum of light. Phys Rev 50, 115-125 (1936).

6. Lin C L, Wang I, Dollet B, Baldeck $\mathrm{P}$ L. Velocimetry microsensors driven by linearly polarized optical tweezers. Opt Lett 31, 329-331 (2006).

7. Li M M, Yan S H, Yao B L, Liang Y S, Han G X et al. Optical trapping force and torque on spheroidal Rayleigh particles with arbitrary spatial orientations. J Opt Soc Am A 33, 1341-1347 (2016).

8. Liaw J W, Chen $\mathrm{Y} S$, Kuo M K. Rotating Au nanorod and nanowire driven by circularly polarized light. Opt Express 22, 26005-26015 (2014).

9. Liaw J W, Chen Y S, Kuo M K. Maxwell stress induced optical torque upon gold prolate nanospheroid. Appl Phys A 122, 182 (2016).

10. Friese M E J, Enger J, Rubinsztein-Dunlop H, Heckenberg N R. Optical angular-momentum transfer to trapped absorbing particles. Phys Rev A 54, 1593-1596 (1996).

11. Paterson L, MacDonald M P, Arlt J, Sibbett W, Bryant P E et al. Controlled rotation of optically trapped microscopic particles. Science 292, 912-914 (2001).

12. Arita $Y$, Richards J M, Mazilu M, Spalding G C, Skelton Spesyvtseva S E et al. Rotational dynamics and heating of trapped nanovaterite particles. ACS Nano 10, 11505-11510 (2016).

13. Wei S B, Wang D P, Lin J, Yuan X C. Demonstration of orbital angular momentum channel healing using a Fabry-Pérot cavity. Opto-Electron Adv 1, 180006 (2018).

14. Parkin S, Knöner G, Singer W, Nieminen T A, Heckenberg N R et al. Optical torque on microscopic objects. Method Cell Biol 82 , 525-561 (2007).

15. Yang Y, Brimicombe P D, Roberts N W, Dickinson M R, Osipov $M$ et al. Continuously rotating chiral liquid crystal droplets in a linearly polarized laser trap. Opt Express 16, 6877-6882 (2008).

16. Kuhn S, Kosloff A, Stickler B A, Patolsky F, Hornberger K et al. Full rotational control of levitated silicon nanorods. Optica 4, 356-360 (2017).

17. Friese $M E J$, Nieminen $T A$, Heckenberg $N \quad R$, Rubinsztein-Dunlop $\mathrm{H}$. Optical alignment and spinning of laser-trapped microscopic particles. Nature 394, 348-350 (1998).

18. Tong L, Miljković V D, Käll M. Alignment, rotation, and spinning of single plasmonic nanoparticles and nanowires using polarization dependent optical forces. Nano Lett 10, 268-273 (2010).

19. Cao $Y Y$, Song $W H$, Ding $W ~ Q$, Sun $F$ K, Zhu T T. Equilibrium orientations of oblate spheroidal particles in single tightly focused Gaussian beams. Opt Express 22, 18113-18118 (2014).

20. Niziev $\vee \mathrm{G}$, Nesterov $A$ V. Influence of beam polarization on laser cutting efficiency. J Phys D: Appl Phys 32, 1455-1461 (1999).

21. La Porta A, Wang M D. Optical torque wrench: angular trapping, rotation, and torque detection of quartz microparticles. Phys Rev Lett 92, 190801 (2004).

22. Datta S, Das B. Electronic analog of the electro-optic modulator. Appl Phys Lett 56, 665-667 (1990).

23. Cheng J C, Nafie L A, Allen S D, Braunstein A I. Photoelastic modulator for the 0.55-13- $\mu \mathrm{m}$ range. Appl Opt 15, 1960-1965 
(1976).

24. Yamaguchi $R$, Nose $T$, Sato $S$. Liquid crystal polarizers with axially symmetrical properties. Jpn J Appl Phys 28, 1730-1731 (1989).

25. Stalder $M$, Schadt $M$. Linearly polarized light with axial symmetry generated by liquid-crystal polarization converters. Opt Lett 21, 1948-1950 (1996).

26. Provenzano C, Pagliusi P, Cipparrone G. Highly efficient liquid crystal based diffraction grating induced by polarization holograms at the aligning surfaces. Appl Phys Lett 89, 121105 (2006).

27. Moreno I, Davis J A, Ruiz I, Cottrell D M. Decomposition of radially and azimuthally polarized beams using a circular-polarization and vortex-sensing diffraction grating. Opt Express 18, 7173-7183 (2010).

28. Liu M J, Chen J, Zhang Y, Shi Y, Zhao C L et al. Generation of coherence vortex by modulating the correlation structure of random lights. Photon Res 7, 1485-1492 (2019).

29. Xiao F J, Shang W Y, Zhu W R, Han L et al., Cylindrical vector beam-excited frequency-tunable second harmonic generation in a plasmonic octamer. Photon Res 6, 157-161 (2018).

30. Chen $\mathrm{R} \mathrm{S}$, Wang $\mathrm{J} \mathrm{H}$, Zhang $X \mathrm{Q}$, Yao $\mathrm{J} \mathrm{N}$, Ming $\mathrm{H}$ et al. Fiber-based mode converter for generating optical vortex beams. Opto-Electron Adv 1, 180003 (2018).

31. Donnay J D H, Donnay G. Optical determination of water content in spherulitic vaterite. Acta Cryst 22, 312-314 (1967).

32. Tracy $S \mathrm{~L}$, Williams $D$ A, Jennings $H M$. The growth of calcite spherulites from solution: II. Kinetics of formation. J Cryst Growth 193, 382-388 (1998)

33. Parkin S J, Vogel R, Persson M, Funk M, Loke $V \mathrm{~L} Y$ et al. Highly birefringent vaterite microspheres: production, characterization and applications for optical micromanipulation. Opt Express 17, 21944-21955 (2009).

34. Bishop A I, Nieminen T A, Heckenberg N R, Rubinsztein-Dunlop $\mathrm{H}$. Optical microrheology using rotating laser-trapped particles. Phys Rev Lett 92, 198104 (2004).

35. Vogel R, Persson M, Feng C, Parkin S J, Nieminen T A et al. Synthesis and surface modification of birefringent vaterite microspheres. Langmuir 25, 11672-11679 (2009).

36. Nieminen $\mathrm{T}$ A, Rubinsztein-Dunlop $\mathrm{H}$, Heckenberg $\mathrm{N} R$. Calculation and optical measurement of laser trapping forces on non-spherical particles. J Quant Spectrosc Radiat Transf 70, 627-637 (2001).

37. Bonin K D, Kourmanov B, Walker T G. Light torque nanocontrol, nanomotors and nanorockers. Opt Express 10, 984-989 (2002).
38. Nieminen T A, Heckenberg N R, Rubinsztein-Dunlop H. Optical measurement of microscopic torques. J Mod Opt 48, 405-413 (2001).

39. Fei P, Nie J, Lee J, Ding Y C, Li S R et al. Subvoxel light-sheet microscopy for high-resolution high-throughput volumetric imaging of large biomedical specimens. Adv Photon 1, 016002 (2019).

40. Li J J, Matlock A C, Li Y Z, Chen Q, Zuo C et al. High-speed in vitro intensity diffraction tomography. Adv Photon 1, 066004 (2019).

41. Feng $S \mathrm{~J}$, Chen $\mathrm{Q}, \mathrm{Gu} \mathrm{G} \mathrm{H}$, Tao $\mathrm{T} Y$, Zhang $L$ et al. Fringe pattern analysis using deep learning. Adv Photon 1, 025001 (2019).

42. Wang $\mathrm{H} \mathrm{Y,} \mathrm{Zheng} \mathrm{J,} \mathrm{Fu} \mathrm{Y} \mathrm{F,} \mathrm{Wang} C$ L, Huang $X \mathrm{R}$ et al. Multichannel high extinction ratio polarized beam splitters based on metasurfaces. Chin Opt Lett 17, 052303 (2019).

43. Rocco D, Gili V F, Ghirardini L, Carletti L, Favero I et al. Tuning the second-harmonic generation in $\mathrm{AIGaAs}$ nanodimers via non-radiative state optimization [Invited]. Photon Res 6, B6-B12 (2018).

44. Nodal Stevens D J, Ávila B J, Rodríguez-Lara B M. Necklaces of PT-symmetric dimers. Photon Res 6, A31-A37 (2018).

45. Sun S, Zhang C, Zhang H T, Gao Y S, Yi N B et al. Enhancing magnetic dipole emission with magnetic metamaterials. Chin Opt Lett 16, 050008 (2018).

46. Liu C, Chen L, Wu T S, Liu Y M, Li J et al. All-dielectric three-element transmissive Huygens' metasurface performing anomalous refraction. Photon Res 7, 1501-1510 (2019).

\section{Acknowledgements}

The work was supported by grants from the National Natural Science Foundation of China (91750203 and 91850111), State Key Laboratory of Applied Optics, Changchun Institute of Optics, Fine Mechanics and Physics, Chinese Academy of Sciences and the High-performance Computing Platform of Peking University.

\section{Competing interests}

The authors declare no competing financial interests.

\section{Supplementary information}

Supplementary information for this paper is available at https://doi.org/10.29026/oea.2020.200022 